\title{
Effect of Poly(epichlorhydrin) on the Crystallization and Compatibility Behavior of Poly(ethylene oxide)/Polyphosphazene Blends
}

\author{
Consuelo R. Herrero and Jose Luis Acosta \\ Instituto de Ciencia y Tecnología de Polímeros \\ C) Juan de la Cierva, 328006 Madrid (Spain)
}

(Received August 9, 1993)

\begin{abstract}
In this research a study was conducted with the aim of gaining insight into the effect exerted by poly(epichlorhydrin) (PECH) on the compatibility and microstructure of the blend consisting of poly(ethylene oxide) (PEO) and a polyphosphazene (PPz). For the different PEO/PPz systems and varying amounts of $\mathrm{PECH}$ isothermal crystallization was analyzed, as well as non-isothermal crystallization, spherulite growth geometry, and the Flory-Huggins interaction parameter. The results obtained point towards a partially compatibilizing effect of $\mathrm{PECH}$ on $\mathrm{PEO} / \mathrm{PPz}$.
\end{abstract}

KEY WORDS Poly(ethylene oxide) / Polyphosphazene / Poly(epichlorohydrin) / Crystallization / Glass Transition Temperature / Spherulite Growth Rate / Compatibility /

According to our findings in previous work, ${ }^{1}$ the blend between poly(ethylene oxide) (PEO) and poly(octafluoropentoxytrifluoroethoxy) phosphazene $(\mathrm{PPz})$ proved to be incompatible over the whole range of compositions. In pursuance of our interest in the synthesis of polymeric electrolytes resulting from solvating the polymeric blend with lithium salts, one of the objective set for this research was to attempt a compatibilization of this polymer blend by means of incorporating another polymer which would act as a compatibilizer, with the effect that ion transfer through the resulting blend would no longer be hindered or interfered with by the presence of interphases.

In this sense poly(epichlorohydrin) (PECH) was chosen as a compatibilizing polymer to combine with the different compositions of the binary system $\mathrm{PEO} / \mathrm{PPz}$. The main interest of our work focused on gaining insight into the microstructure and compatibility of the system PEO/PPz. In the course of this study the crystallization kinetics was analyzed, glass transition temperature was measured, spherulite growth kinetics was recorded and the FloryHuggins interaction parameter was determined via $\mathrm{PEO}$ melting point depression.

\section{EXPERIMENTAL}

The following commercial polymers were used: Poly(ethylene oxide) (PEO) $\left(M_{w}=5.0 \times\right.$ $10^{6}$ ) was supplied by Aldrich; the poly(octafluoropentoxytrifluoroethoxy) phosphazene (PPz) PNF-200 was obtained from Firestone and the poly(epichlorohydrin) $(\mathrm{PECH})\left(M_{w}=\right.$ $7.0 \times 10^{5}$ ) from Aldrich.

The blends were obtained by dissolving in acetonitrile and chloroform the already proportioned polymers, subsequently vaporizing the solvent and drying the samples under high vacuum conditions until constant sample weight was achieved.

Isothermal crystallization and melting were measured in a Perkin Elmer differential scanning calorimeter DSC 7. The samples were heated to $100^{\circ} \mathrm{C}$, where they were held for five minutes in order to delete their thermal 
antecedents and then quenched $\left(350^{\circ} \mathrm{C} \mathrm{min}^{-1}\right)$ down to their crystallization temperature $T_{\mathrm{c}}$. When they had completely crystallized, the samples were brought to melt at a rate of $10^{\circ} \mathrm{C} \mathrm{min}^{-1}$.

Non-isothermal crystallization was studied by recording the respective non-isothermal crystallization thermograms in a Mettler differential scanning calorimeter TA 4000 at rates of $20,10,5,2$, and $1^{\circ} \mathrm{C} \mathrm{min}{ }^{-1}$.

The glass transition temperature $T_{\mathrm{g}}$ was measured in a Mettler differential scanning calorimeter TA 4000. The samples remained at $100^{\circ} \mathrm{C}$ for 5 minutes and were then quenched down to $-100^{\circ} \mathrm{C}$, their thermograms being subsequently recorded at a rate of $10^{\circ} \mathrm{Cmin}^{-1}$.

Spherulite growth was monitored under a Jenaval microscope coupled to a Hot Stage Mettler FP82. The samples were heated to $100^{\circ} \mathrm{C}$ for 5 minutes and then quenched to their $T_{\mathrm{c}}$, where the radii were measured. The tridimensional (3D) graphs were obtained with GOLDEN graphics software.

\section{RESULTS AND DISCUSSION}

Study of the Microstructure and Morphology of the PEO/PPz, PEO/PECH, and PEO/PPz| PECH Blends

The isothermal crystallization kinetics of all the experimental systems were analyzed applying Avrami's equation ${ }^{2}$

$$
X_{t}=1-\exp \left(-K_{t}^{n}\right)
$$

where $X_{t}$ is the weight fraction of the crystallizable material at time $t, K$ stands for the rate constant, and $n$ is the Avrami exponent, which depends on the type of nucleation and the growth geometry of the crystals.

The values of $K$ and $n$ are obtained for each crystallization temperature from the $\mathrm{Y}$-intercept and the slope, respectively, of the plot of $\log \left(-\ln \left(1-X_{t}\right)\right) v s . \log t$.

In Tables I-III the results are compiled as obtained from the study of the crystallization kinetics of the systems PEO/PPz, PEO/PECH,
Table I. Isothermal crystallization of the $\mathrm{PEO} / \mathrm{PPz}$ system

\begin{tabular}{|c|c|c|c|c|}
\hline $\begin{array}{c}\text { Blend } \\
\text { composition } \\
\mathrm{PEO} / \mathrm{PPz}\end{array}$ & $\frac{T_{\mathrm{c}}}{{ }^{\circ} \mathrm{C}}$ & $n$ & $\frac{\log K}{\min ^{-n}}$ & $\frac{t_{1 / 2}}{\min }$ \\
\hline \multirow[t]{3}{*}{$100 / 0$} & 44 & 2.427 & -0.433 & 1.298 \\
\hline & 46 & 2.405 & -0.730 & 1.727 \\
\hline & 48 & 2.543 & -2.515 & 8.439 \\
\hline \multirow[t]{3}{*}{$80 / 20$} & 44 & 1.945 & -0.013 & 0.841 \\
\hline & 46 & 1.949 & -1.060 & 2.898 \\
\hline & 48 & 2.110 & -2.423 & 11.820 \\
\hline \multirow[t]{3}{*}{$60 / 40$} & 44 & 2.807 & -0.103 & 0.955 \\
\hline & 46 & 2.081 & -0.369 & 1.262 \\
\hline & 48 & 2.178 & -1.176 & 2.967 \\
\hline \multirow[t]{3}{*}{$40 / 60$} & 44 & 2.256 & -0.413 & 1.297 \\
\hline & 46 & 2.328 & -1.380 & 3.349 \\
\hline & 48 & 2.259 & -2.359 & 9.435 \\
\hline \multirow[t]{3}{*}{$20 / 80$} & 44 & 2.080 & -0.560 & 1.556 \\
\hline & 46 & 2.070 & -1.390 & 3.942 \\
\hline & 48 & 2.696 & -3.240 & 13.940 \\
\hline
\end{tabular}

Table II. Isothermal crystallization of the PEO/PECH system

\begin{tabular}{|c|c|c|c|c|}
\hline \multirow{2}{*}{$\begin{array}{c}\text { Blend } \\
\text { composition } \\
\text { PEO/PECH }\end{array}$} & $T_{\mathrm{c}}$ & \multirow{2}{*}{$n$} & \multirow{2}{*}{$\frac{\log K}{\min ^{-n}}$} & \multirow{2}{*}{$\frac{t_{1 / 2}}{\min }$} \\
\hline & ${ }^{\circ} \mathrm{C}$ & & & \\
\hline \multirow[t]{3}{*}{$100 / 0$} & 44 & 2.427 & -0.433 & 1.298 \\
\hline & 46 & 2.405 & -0.730 & 1.727 \\
\hline & 48 & 2.543 & -2.515 & 8.439 \\
\hline \multirow[t]{3}{*}{$80 / 20$} & 44 & 2.222 & -1.491 & 1.659 \\
\hline & 46 & 2.599 & -2.969 & 2.722 \\
\hline & 48 & 2.409 & -4.103 & 4.716 \\
\hline \multirow[t]{3}{*}{$60 / 40$} & 40 & 2.359 & -1.230 & 1.442 \\
\hline & 46 & 2.462 & -2.010 & 1.949 \\
\hline & 44 & 2.618 & -3.202 & 2.953 \\
\hline \multirow[t]{3}{*}{$40 / 60$} & 40 & 2.546 & -4.136 & 4.393 \\
\hline & 42 & 2.343 & -5.246 & 6.403 \\
\hline & 44 & 2.242 & -5.354 & 9.252 \\
\hline
\end{tabular}

as well as that of the ternary system PEO/ $\mathrm{PPz} / \mathrm{PECH}$. Figure 1 shows the logarithm of the rate constant $(\log K)$ plotted against blend composition at a crystallization temperature of $44^{\circ} \mathrm{C}$ for the binary systems $\mathrm{PEO} / \mathrm{PPz}$ and $\mathrm{PEO} / \mathrm{PECH}$, whereas Figure 2 represents the response surface of the PEO rate constant at $44^{\circ} \mathrm{C}$, when contained in ternary blends with $\mathrm{PPz}$ and $\mathrm{PECH}$. As can be observed in Figure 
A

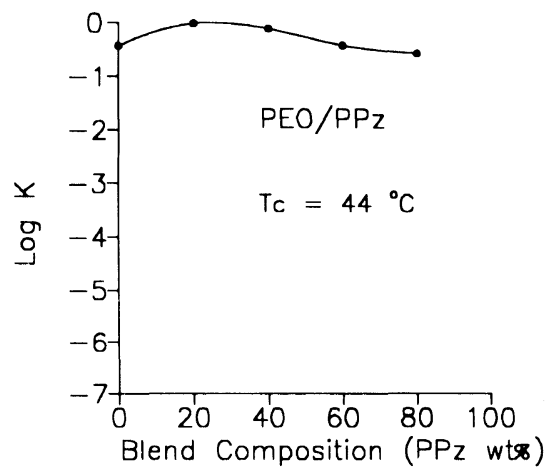

B

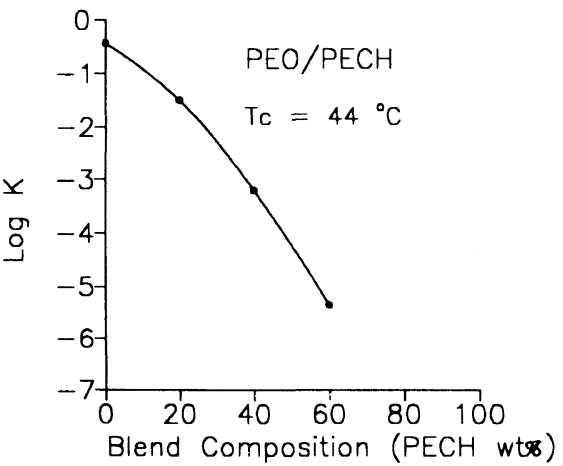

Figure 1. Effect of the PPz or PECH contents on the isothermal overall kinetic rate constant $\log K$ crystallized at $44^{\circ} \mathrm{C}$.

Table III. Isothermal crystallization of the $\mathrm{PEO} / \mathrm{PPz} / \mathrm{PECH}$ system

\begin{tabular}{|c|c|c|c|c|}
\hline \multirow{2}{*}{$\begin{array}{c}\text { Blend } \\
\text { composition } \\
\mathrm{PEO} / \mathrm{PPz} / \mathrm{PECH}\end{array}$} & $T_{\mathrm{c}}$ & \multirow{2}{*}{$n$} & \multirow{2}{*}{$\frac{\log K}{\min ^{-n}}$} & \multirow{2}{*}{$\frac{t_{1 / 2}}{\min }$} \\
\hline & ${ }^{\circ} \mathrm{C}$ & & & \\
\hline \multirow[t]{3}{*}{$100 / 0 / 0$} & 44 & 2.427 & -0.433 & 1.298 \\
\hline & 46 & 2.405 & -0.730 & 1.727 \\
\hline & 48 & 2.543 & -2.515 & 8.439 \\
\hline \multirow[t]{3}{*}{$60 / 20 / 20$} & 44 & 1.947 & -2.330 & 2.740 \\
\hline & 46 & 1.766 & -2.817 & 4.007 \\
\hline & 48 & 1.817 & -3.587 & 5.883 \\
\hline \multirow[t]{3}{*}{$20 / 60 / 20$} & 44 & 2.108 & -1.457 & 1.689 \\
\hline & 46 & 1.682 & -1.612 & 2.096 \\
\hline & 48 & 1.649 & -2.207 & 3.053 \\
\hline \multirow[t]{3}{*}{$20 / 20 / 60$} & 44 & 2.077 & -0.472 & 1.052 \\
\hline & 46 & 1.801 & -1.333 & 1.711 \\
\hline & 48 & 1.603 & -2.080 & 2.911 \\
\hline \multirow[t]{3}{*}{$33.3 / 33.3 / 33.3$} & 44 & 1.772 & -1.626 & 2.036 \\
\hline & 46 & 1.554 & -2.194 & 3.243 \\
\hline & 48 & 1.841 & -3.221 & 4.715 \\
\hline
\end{tabular}

1A, the PEO crystallization rate constant remains practically the same for all $\mathrm{PPz}$ concentrations, as is characteristic of an incompatible system. In Figure 1B, however, which corresponds to the binary system PEO/ $\mathrm{PECH}$, the rate constant diminishes inversely proportionate to $\mathrm{PECH}$ concentration, which is indicative of the compatible nature of the system. In Figure 2 (ternary system), in contrast, two clearly differentiated effects are discernible for each $\mathrm{PEO} / \mathrm{PPz}$ concentration;

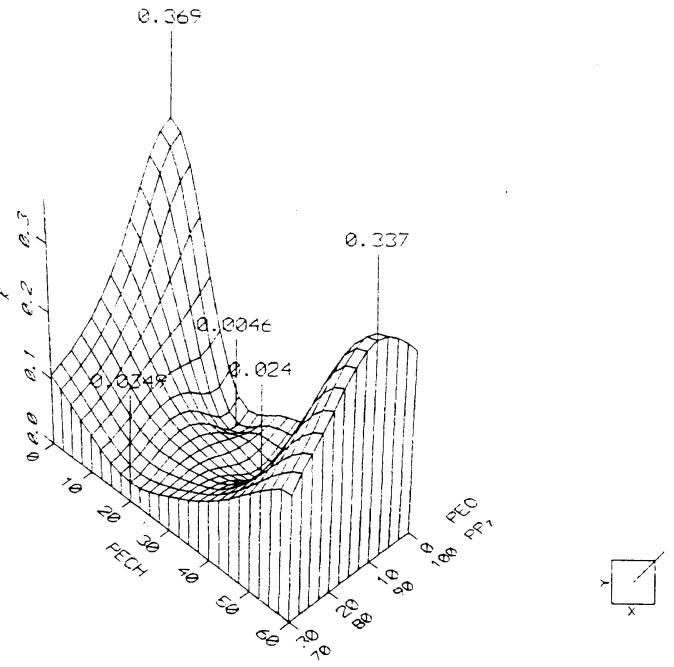

Figure 2. Effect of the PECH on the isothermal overall kinetic rate constant $K$ of the $\mathrm{PEO} / \mathrm{PPz}$ blend crystallized at $44^{\circ} \mathrm{C}$.

there is an initial section, where with increasing $\mathrm{PECH}$ portion in the system the PEO rate constant diminishes. As of $c a .20 \% \mathrm{PECH}$ concentration this behavior is reversed, and the PEO rate constant grows with all subsequent $\mathrm{PECH}$ increments in the blend. This behaviour is typical of binary or ternary systems in which one of the polymers, apart from acting as a dilutant vis-à-vis the other (in our case this role is played by $\mathrm{PECH}$ ), exerts a highly prominent nucleating effect on the 
crystallization of the crystalline polymer.

The PEO crystal growth geometry, as inferred from the Avrami exponent $(n)$, is not affected by the presence of neither $\mathrm{PPz}$ nor $\mathrm{PECH}$ in the case of binary blends, considering that constancy of $n$ suggests a growth geometry intermediate between bi- and tri-dimensionality. In the case of ternary composites, there coexist two distinct growth geometries, one monodimensional, and the other bidimensional, as can be inferred from the values of $n$ in Table I-III, in clear contrast to the binary systems.

The PEO non-sothermal crystallization kinetics was analyzed applying Avrami's method extended to non-isothermal conditions, ${ }^{2}$ as well as the Ozawa method. ${ }^{3}$

In the first case, Avrami's equation (eq 1) was directly applied to the data obtained from the non-isothermal crystallization thermograms. The plot of $\log (-\ln (1-\alpha(t))) v s . \log t$ for each crystallization rate yielded ever so many straight lines, from whose slopes the values of $n$ were determined, whereas the values of $K$ were taken from the respective $\mathrm{Y}$ intercepts. In non-isothermal crystallization the values of $n$ and $K$ do not have the same meaning as in isothermal crystallization, due to the fact that under non-isothermal conditions the temperature changes constantly, and hence spherulite growth rate and nucleation are temperature-dependent.

The Ozawa method takes Avrami's equation as a starting point, yet modifies it conveniently by means of the temperature function, ${ }^{4}$ thus providing an equation capable of describing

Table IV. $K$ and $n$ parameters for non-isothermal crystallization of $\mathrm{PEO} / \mathrm{PPz}$

\begin{tabular}{|c|c|c|c|c|c|c|}
\hline \multirow{3}{*}{$\begin{array}{c}\text { Blend composition } \\
\mathrm{PEO} / \mathrm{PPz}\end{array}$} & \multicolumn{3}{|c|}{ Ozawa } & \multicolumn{3}{|c|}{ Avrami } \\
\hline & $T_{\mathrm{c}}$ & & $K$ & Rate & & $K$ \\
\hline & ${ }^{\circ} \mathrm{C}$ & & $\min ^{-n}$ & ${ }^{\circ} \mathrm{C} \min ^{-1}$ & & $\min ^{-n}$ \\
\hline \multirow[t]{5}{*}{$100 / 0$} & 36 & 0.892 & 1.745 & 20 & 1.808 & 1.416 \\
\hline & 38 & 1.022 & 1.653 & 10 & 1.882 & 1.399 \\
\hline & 40 & 1.234 & 1.610 & 5 & 2.059 & 0.239 \\
\hline & 42 & 1.430 & 1.446 & 2 & 2.053 & 0.052 \\
\hline & 44 & .1 .624 & 1.059 & 1 & 2.356 & 0.011 \\
\hline \multirow[t]{5}{*}{$80 / 20$} & 40 & 1.142 & 2.294 & 20 & 1.807 & 1.706 \\
\hline & 42 & 1.175 & 1.824 & 10 & 1.870 & 0.778 \\
\hline & 44 & 1.313 & 1.515 & 5 & 1.854 & 0.406 \\
\hline & 46 & 1.413 & 0.909 & 2 & 2.082 & 0.086 \\
\hline & & & & 1 & 2.664 & 0.008 \\
\hline \multirow[t]{5}{*}{$60 / 40$} & 40 & 1.209 & 2.472 & 20 & 2.041 & 1.637 \\
\hline & 42 & 1.037 & 2.010 & 10 & 2.046 & 0.731 \\
\hline & 44 & 0.924 & 1.280 & 5 & 2.003 & 0.276 \\
\hline & 46 & 1.400 & 1.398 & 2 & 2.332 & 0.064 \\
\hline & 48 & 1.813 & 1.055 & 1 & 2.107 & 0.035 \\
\hline \multirow[t]{5}{*}{$40 / 60$} & 40 & 1.483 & 2.645 & 20 & 2.110 & 1.603 \\
\hline & 42 & 1.734 & 2.437 & 10 & 2.257 & 0.668 \\
\hline & 44 & 1.691 & 1.476 & 5 & 2.447 & 0.262 \\
\hline & 46 & 1.542 & 0.638 & 2 & 2.382 & 0.058 \\
\hline & & & & 1 & 2.546 & 0.014 \\
\hline \multirow[t]{5}{*}{$20 / 80$} & 40 & 2.098 & 3.369 & 20 & 2.078 & 2.999 \\
\hline & 42 & 2.198 & 2.893 & 10 & 2.100 & 1.172 \\
\hline & 44 & 2.200 & 1.706 & 5 & 2.693 & 0.475 \\
\hline & 46 & 3.028 & 0.789 & 2 & 3.486 & 0.038 \\
\hline & & & & 1 & 2.642 & 0.047 \\
\hline
\end{tabular}


Table V. $K$ and $n$ parameters for non-isothermal crystallization of PEO/PECH.

\begin{tabular}{|c|c|c|c|c|c|c|}
\hline \multirow{3}{*}{$\begin{array}{l}\text { Blend composition } \\
\text { PEO/PECH }\end{array}$} & \multicolumn{3}{|c|}{ Ozawa } & \multicolumn{3}{|c|}{ Avrami } \\
\hline & $T_{\mathrm{c}}$ & & $K$ & Rate & & $K$ \\
\hline & ${ }^{\circ} \mathrm{C}$ & & $\min ^{-n}$ & ${ }^{\circ} \mathrm{C} \min ^{-1}$ & & $\min ^{-n}$ \\
\hline \multirow[t]{5}{*}{$100 / 0$} & 36 & 0.892 & 1.745 & 20 & 1.808 & 1.416 \\
\hline & 38 & 1.022 & 1.653 & 10 & 1.882 & 1.399 \\
\hline & 40 & 1.234 & 1.610 & 5 & 2.059 & 0.239 \\
\hline & 42 & 1.430 & 1.446 & 2 & 2.053 & 0.052 \\
\hline & 44 & 1.624 & 1.059 & 1 & 2.356 & 0.011 \\
\hline \multirow[t]{5}{*}{$80 / 20$} & 34 & 0.768 & 2.000 & 20 & 2.219 & 1.489 \\
\hline & 36 & 0.913 & 1.951 & 10 & 2.403 & 0.616 \\
\hline & 38 & 1.022 & 1.744 & 5 & 2.345 & 0.366 \\
\hline & 40 & 1.263 & 1.609 & 2 & 2.944 & 0.036 \\
\hline & 42 & 1.797 & 1.499 & 1 & 3.271 & 0.006 \\
\hline \multirow[t]{5}{*}{$60 / 40$} & 32 & 1.509 & 3.054 & 20 & 2.276 & 1.956 \\
\hline & 34 & 2.937 & 1.681 & 10 & 2.492 & 0.823 \\
\hline & 36 & 2.419 & 3.396 & 5 & 2.792 & 0.266 \\
\hline & 38 & 2.690 & 1.897 & 2 & 3.167 & 0.031 \\
\hline & & & & 1 & 3.696 & 0.002 \\
\hline \multirow[t]{5}{*}{$40 / 60$} & 24 & 2.465 & 3.997 & 20 & 2.780 & 1.387 \\
\hline & 26 & 2.965 & 4.137 & 10 & 3.423 & 0.326 \\
\hline & 28 & 2.076 & 2.082 & 5 & 3.725 & 0.058 \\
\hline & 30 & 2.602 & 1.819 & 2 & 3.734 & 0.005 \\
\hline & 32 & 3.338 & 1.404 & 1 & 3.125 & 0.002 \\
\hline
\end{tabular}

non-isothermal crystallization in a semicrystalline polymer:

$$
\begin{gathered}
\ln (-\ln (1-\alpha(t)))=\ln X(T)-n \ln \beta \\
X(T)=n A \int_{T_{0}}^{T} \exp \left(\frac{E^{\prime}}{T}\right)\left(T-T_{0}\right)^{n-1} \mathrm{~d} T
\end{gathered}
$$

where $E^{\prime}=E / R$ and $\beta$ is the cooling rate.

Applying eq 2 the Avrami exponent is determined as a function of the cooling rate and the crystallized polymer fraction $\alpha(t)$.

Tables IV-VI compile de values of the Avrami exponent $n$ and those of $K$, as obtained from applying the Avrami and the Ozawa methods both to binary to ternary systems, Figures 3 and 4 show Avrami rate constant $K$ plotted against amorphous polymer concentration as obtained by applying both the Avrami and the Ozawa method to non-isothermal conditions. In Figure $3 \mathrm{~A}$ it can be observed that $K$ grows with increasing $\mathrm{PECH}$ concentra- tion, as is typical for compatible systems, whereas in the case of the blend $\mathrm{PEO} / \mathrm{PPz}$ (Figure 4A) the $K$ value of $\mathrm{PEO}$ remains practically invariable when increasing the $\mathrm{PPz}$ portion in the blend, this latter effect becoming the more prominent the higher the crystallization rates. This behavior identifies an incompatible system, where the PEO crystals grow in their own melt and remain unaffected by the presence of another polymer. In Figures 3B and 4B the Ozawa $K$ values are plotted against the $\mathrm{PECH}$ and $\mathrm{PPz}$ concentrations, respectively. $K$ is shown to increase with increasing amorphous polymer portion. This effect, however, is stronger in the case of $\mathrm{PECH}$, this confirming the compatibility existing in the case of PEO/PECH blends and the only partial compatibility in the case of PEO/PPz. Regarding the $K$ of PEO in the ternary blends (Table VI, Figure 6), for each rate and blend composition, $K$ is observed to diminish 
Microstructure of $\mathrm{PEO} / \mathrm{PPz} / \mathrm{PECH}$ Blends

Table VI. $K$ and $n$ parameters for non-isothermal crystallization of PEO/PPz/PECH

\begin{tabular}{|c|c|c|c|c|c|c|}
\hline \multirow{3}{*}{$\begin{array}{l}\text { Blend composition } \\
\mathrm{PEO} / \mathrm{PPz} / \mathrm{PECH}\end{array}$} & \multicolumn{3}{|c|}{ Ozawa } & \multicolumn{3}{|c|}{ Avrami } \\
\hline & $T_{\mathrm{c}}$ & & $K$ & Rate & & $K$ \\
\hline & ${ }^{\circ} \mathrm{C}$ & & $\min ^{-n}$ & ${ }^{\circ} \mathrm{C} \min ^{-1}$ & & $\min ^{-n}$ \\
\hline \multirow[t]{5}{*}{$100 / 0 / 0$} & 36 & 0.892 & 1.745 & 20 & 1.808 & 1.416 \\
\hline & 38 & 1.022 & 1.653 & 10 & 1.882 & 1.399 \\
\hline & 40 & 1.234 & 1.610 & 5 & 2.059 & 0.239 \\
\hline & 42 & 1.430 & 1.446 & 2 & 2.053 & 0.052 \\
\hline & 44 & 1.624 & 1.059 & 1 & 2.356 & 0.011 \\
\hline \multirow[t]{5}{*}{$60 / 20 / 20$} & 32 & 1.227 & 2.590 & 20 & 2.056 & 1.312 \\
\hline & 34 & 1.347 & 2.478 & 10 & 2.150 & 0.469 \\
\hline & 36 & 1.562 & 2.438 & 5 & 2.386 & 0.138 \\
\hline & 38 & 1.686 & 3.170 & 2 & 2.337 & 0.034 \\
\hline & 40 & 1.365 & 1.067 & 1 & 2.571 & 0.005 \\
\hline \multirow[t]{5}{*}{$20 / 60 / 20$} & 34 & 1.402 & 2.774 & 20 & 2.871 & 1.977 \\
\hline & 36 & 1.518 & 2.341 & 10 & 3.305 & 0.456 \\
\hline & 38 & 1.962 & 2.277 & 5 & 3.307 & 0.107 \\
\hline & 40 & 2.480 & 2.050 & 2 & 3.863 & 0.003 \\
\hline & & & & 1 & 3.551 & 0.001 \\
\hline \multirow[t]{5}{*}{$20 / 20 / 60$} & 28 & 0.464 & 0.794 & 20 & 2.123 & 1.134 \\
\hline & 30 & 0.573 & 0.771 & 10 & 2.072 & 0.314 \\
\hline & 32 & 0.709 & 0.405 & 5 & 1.588 & 0.164 \\
\hline & 34 & 0.911 & 0.308 & 2 & 1.726 & 0.026 \\
\hline & 36 & 0.603 & 0.071 & 1 & 1.696 & 0.011 \\
\hline \multirow[t]{5}{*}{$33.3 / 33.3 / 33.3$} & 32 & 1.478 & 1.912 & 20 & 2.444 & 0.649 \\
\hline & 34 & 1.557 & 1.419 & 10 & 2.633 & 0.173 \\
\hline & 36 & 1.141 & 0.673 & 5 & 2.856 & 0.033 \\
\hline & 38 & 1.675 & 0.827 & 2 & 2.429 & 0.013 \\
\hline & & & & 1 & 2.006 & 0.013 \\
\hline
\end{tabular}
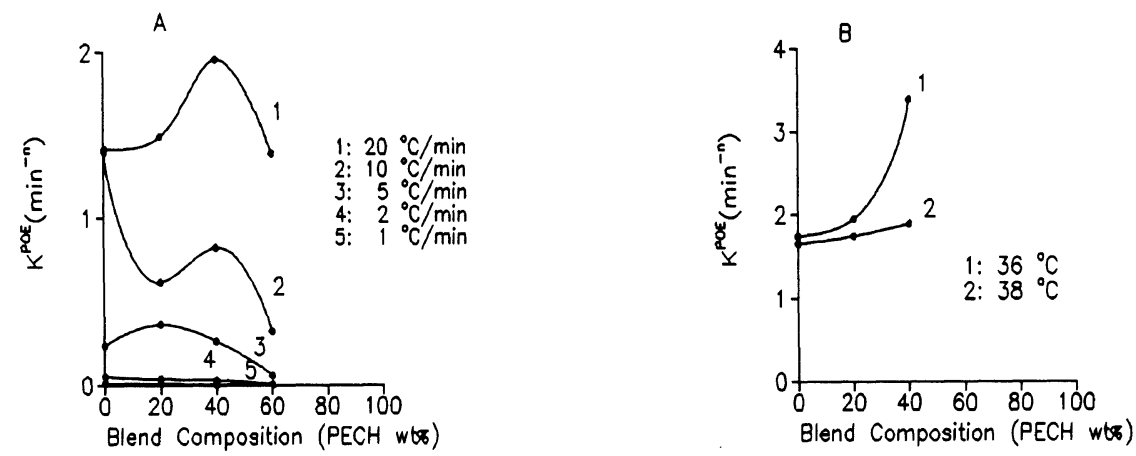

Figure 3. Effect of the PECH on the non-isothermal rate constant using A) Avrami method and B) Ozawa method.

inversely proportionate to $\mathrm{PECH}$ concentration, de decrease becoming the more prominent the greater the $\mathrm{PECH}$ portion in the blend, an identical regularity having been observed for isothermal conditions. The same phenomenon became apparent, when $K$ was analyzed in 
A

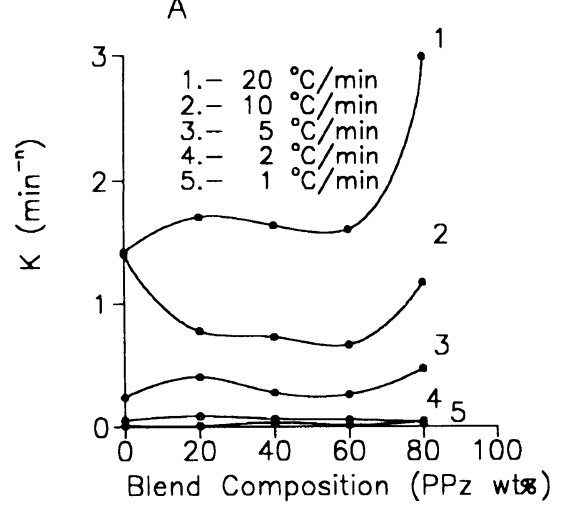

B

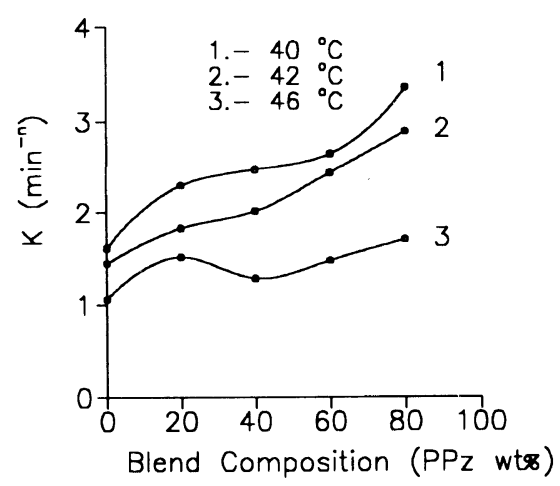

Figure 4. Effect of the $\mathrm{PPz}$ on the non-isothermal rate constant using A) Avrami method and B) Ozawa method.

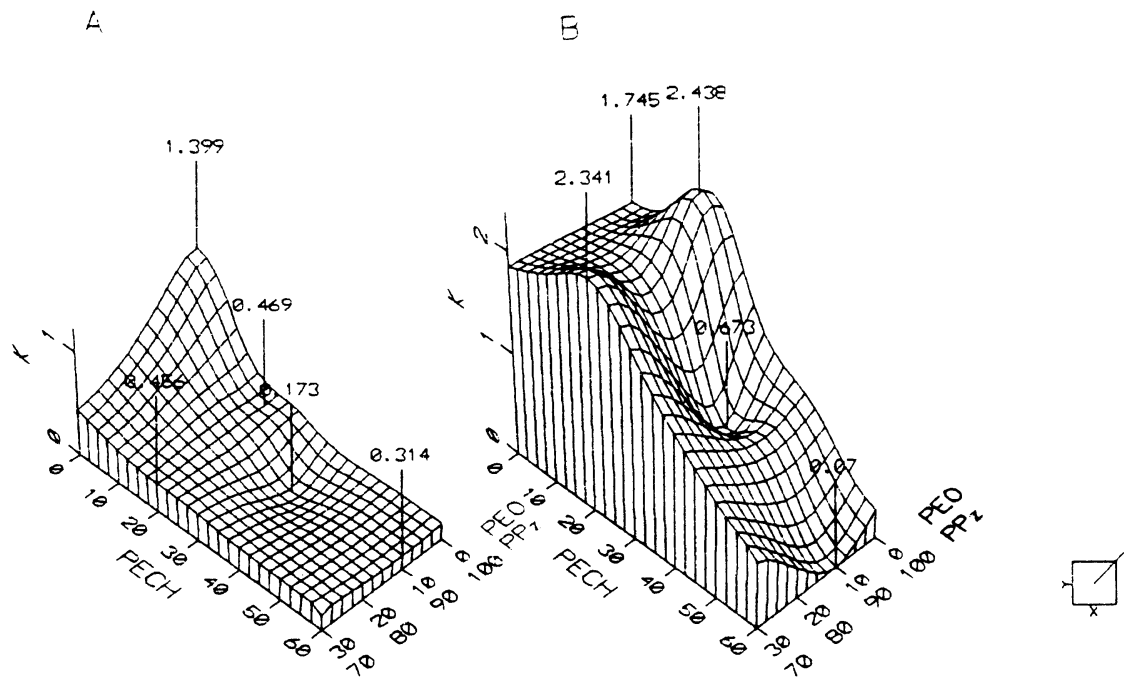

Figure 5. Effect of the $\mathrm{PECH}$ on the non-isothermal rate constant of the $\mathrm{PEO} / \mathrm{PPz}$ blend using $\mathrm{A}$ ) Avrami method and B) Ozawa method.

terms of the Ozawa method (Figure 5B), although the values obtained were, on the whole, slightly higher than those calculated with the Avrami approach. These findings allow for the interference that PECH exerts a diluting effect, both when in the presence of $\mathrm{PEO}$ or in combination with $\mathrm{PEO} / \mathrm{PPz}$. Yet full $\mathrm{PEO} / \mathrm{PPz}$ compatibilization is not achieved by the presence of PECH.

Under non-isothermal conditions the values of the Avrami exponent, as a general rule, are observed to be higher than those computed for isothermal conditions. Yet opposite is observed, when the $n$ values are obtained via the Ozawa method; they are always lower than the Avrami values, both for isothermal and non-isothermal conditions (Tables IV-VI).

\section{Spherulite Growth}

PEO presents a spherulite structure when 
crystallized from a melt. ${ }^{5,6}$ The plot of the spherulite growth rate $v s$. blend composition is reproduced in Figure 6, which shows that, when the system $\mathrm{PEO} / \mathrm{PPz}$ crystallizes in the presence of $\mathrm{PECH}$, the spherulite growth rate increases together with $\mathrm{PPz}$ increments (basically incompatible systems). In the presence of $\mathrm{PECH}$, however, the growth rate diminishes, which is behavior to be expected of a compatible systems. As these behaviors are directly related to nucleation density and the nucleus formation energy, we determined these latter parameters in terms of general theory, in order to relate them to blend composition and

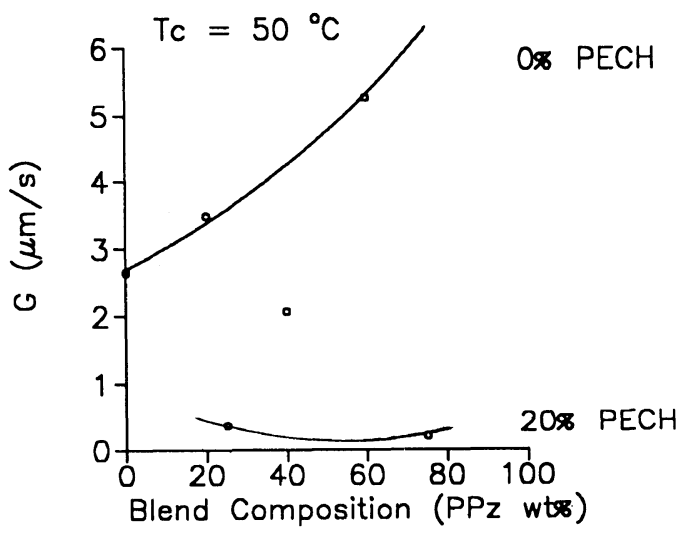

Figure 6. Variation of the spherulite growth rate with the concentration at different PECH composition. compatibility.

The nucleus formation energy relates to the nucleation factor $K_{\mathrm{g}}$, which is obtained from the equation describing spherulite growth rate for crystalline polymers when containing a diluting polymer. ${ }^{7-9}$

The spherulite growth rate for dilutant polymer blends can be expressed through the following equation:

$$
\begin{aligned}
\alpha & =\ln G-\ln v_{2}+\frac{U^{*}}{R\left(T_{\mathrm{c}}-T_{\mathrm{g}}+C\right)}-\frac{0.2 T_{\mathrm{m}} \ln v_{2}}{\Delta T} \\
& =\ln G_{0}-\frac{K_{\mathrm{g}}}{T_{\mathrm{c}} \Delta T_{f}}
\end{aligned}
$$

where $G_{0}$ is a constant (pre-exponential factor); $U^{*}=U_{1}{ }^{*}+U_{2}{ }^{*}$ is the sum of activation

Table VII. $K_{\mathrm{g}}$ and nucleation density values of studied blends at $48^{\circ} \mathrm{C}$

\begin{tabular}{ccccc}
\hline $\begin{array}{c}\text { Blend } \\
\text { composition }\end{array}$ & $N \times 10^{3}$ & & $K_{\mathrm{g}} \times 10^{-4}$ & \\
PEO/PPz/PECH & nuclei cm $\mathrm{cm}^{-3}$ & & $\left(\mathrm{~K}^{2}\right)$ & \\
\hline $100 / 0 / 0$ & 0.04 & & 6.928 & \\
\hline $80 / 20 / 0$ & 0.02 & & 5.123 & 28.184 \\
$60 / 40 / 0$ & 3.66 & & 3.196 & 26.955 \\
$40 / 60 / 0$ & - & & 3.066 & 27.178 \\
$80 / 0 / 20$ & 2.33 & & 7.146 & 29.382 \\
$60 / 20 / 20$ & 22.90 & & 8.331 & 32.232 \\
$20 / 60 / 20$ & 882.70 & & 11.138 & 38.141 \\
& & &
\end{tabular}
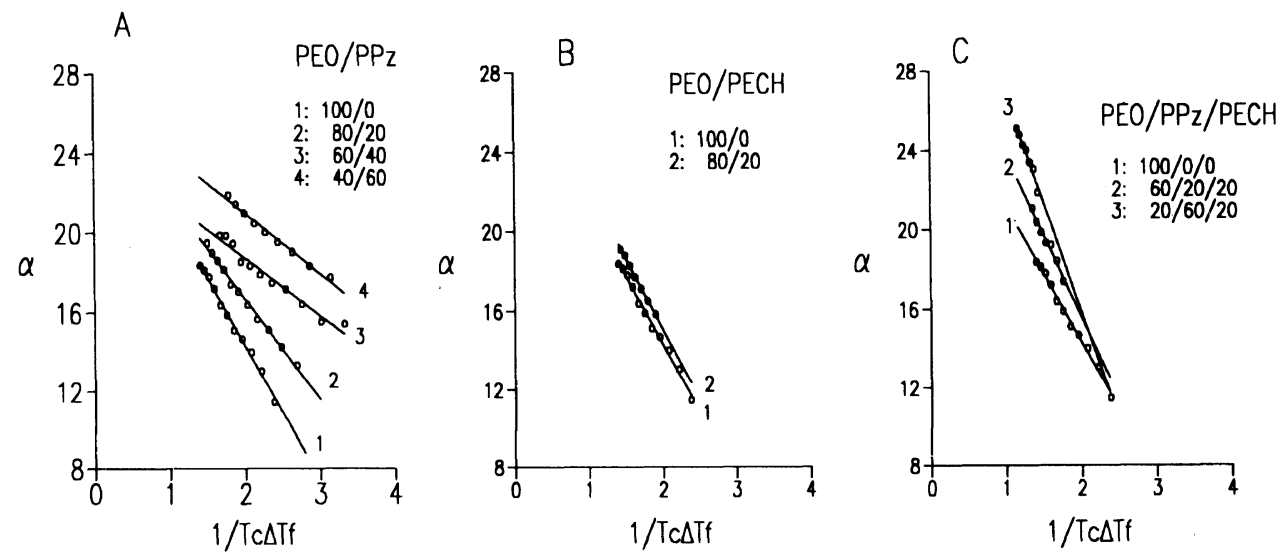

Figure 7. Plots $\alpha$ s. $1 / T_{\mathrm{c}} \Delta T_{\mathrm{f}}$ for A) $\mathrm{PEO} / \mathrm{PPz}$ blend, B) $\mathrm{PEO} / \mathrm{PECH}$ blend and C) $\mathrm{PEO} / \mathrm{PPz} / \mathrm{PECH}$ blend. 
Table VIII. Glass transition temperature values of studied blends

\begin{tabular}{|c|c|c|c|c|c|}
\hline \multicolumn{2}{|c|}{ Blend composition } & \multirow[b]{2}{*}{$T_{\mathrm{g}}^{\text {POE }}$} & \multirow[b]{2}{*}{$T_{\mathrm{g}}^{\text {Blend }}$} & \multirow[b]{2}{*}{$T_{\mathrm{g}}^{\mathrm{PECH}}$} & \multirow[b]{2}{*}{$T_{\mathbf{g}}^{\mathbf{P P z}}$} \\
\hline $\mathrm{PEO} / \mathrm{PPz}$ & PECH & & & & \\
\hline wt $\%$ & $\mathrm{wt} \%$ & ${ }^{\circ} \mathrm{C}$ & ${ }^{\circ} \mathrm{C}$ & ${ }^{\circ} \mathrm{C}$ & ${ }^{\circ} \mathrm{C}$ \\
\hline $100 / 0$ & 0 & - & -52.9 & - & - \\
\hline $80 / 0$ & 20 & - & -47.2 & - & - \\
\hline $60 / 0$ & 40 & - & -30.3 & - & - \\
\hline $40 / 0$ & 60 & - & -36.4 & - & - \\
\hline $20 / 0$ & 80 & - & -30.8 & - & - \\
\hline $100 / 0$ & 0 & -52.9 & - & - & - \\
\hline $80 / 20$ & 0 & -54.6 & - & - & -64.5 \\
\hline $60 / 40$ & 0 & -51.8 & - & - & -62.6 \\
\hline $40 / 60$ & 0 & -51.6 & - & - & -62.2 \\
\hline $20 / 80$ & 0 & -50.5 & - & - & -60.9 \\
\hline $100 / 0$ & 0 & - & - & - & -60.7 \\
\hline $0 / 0$ & 100 & - & - & -23.1 & - \\
\hline $0 / 20$ & 80 & - & - & -23.8 & - \\
\hline $0 / 40$ & 60 & - & - & -23.7 & - \\
\hline $0 / 60$ & 40 & - & - & -23.8 & -63.1 \\
\hline $0 / 80$ & 20 & - & - & -22.6 & -62.0 \\
\hline $60 / 20$ & 20 & - & -36.03 & - & -65.93 \\
\hline $20 / 20$ & 60 & - & -32.90 & - & -63.63 \\
\hline $20 / 60$ & 20 & - & -30.83 & - & -62.47 \\
\hline $33.3 / 33.3$ & 33.3 & - & -35.53 & - & -63.30 \\
\hline
\end{tabular}

energies movement of the crystallizable and non-crystallizable molecules in the melt and has value 4120 for these systems. $T_{\mathrm{g}}$ is the glass transition temperature and $T_{\mathrm{m}}$ the melting temperature. $C$ represents a constant, and $f$ is a factor taking into account the dependence of $\Delta H_{\mathrm{v}}$ on the crystallization temperature, ${ }^{10}$ equivalent to $f=2 T_{\mathrm{c}} /\left(T_{\mathrm{m}}{ }^{0}+T_{\mathrm{c}}\right)$, where $T_{\mathrm{m}}{ }^{0}$ is the thermodynamic melting temperature, $v_{2}$ the volume fraction and $K_{\mathrm{g}}$ the nucleation factor.

Plotting $\alpha$ vs. $1 /\left(T_{\mathrm{c}} \Delta T_{\mathrm{f}}\right)$ (Figure 7) straight lines are obtained whose slopes determine the values of $K_{\mathrm{g}}$, and whose $\mathrm{Y}$-intercepts yeild the values of the pre-exponential factor $G_{0}$, which in our case is $G_{0}=12 \times 10^{-5} \mathrm{~cm} \mathrm{~s}^{-1}$, in reasonable agreement with the value described in the literature. ${ }^{11}$ The values of $K_{\mathrm{g}}$ and $N$ are listed in Table VIII. The nucleation density was determined applying the following expression, which assumes nucleation to be of a heterogeneous and morphology to be spherulitic nature:

$$
K_{n}=\frac{4}{3} \pi N G^{3} \frac{\rho_{\mathrm{c}}}{\rho_{\mathrm{a}}} \frac{1}{1-X_{\mathrm{t}}}
$$

where $G$ is the spherulite growth rate, $K_{n}$ the crystallization growth rate constant (as obtained from the expression $K_{n}=\ln 2 /\left(t_{1 / 2}\right)^{n}(13)$ where $n$ is the Avrami exponent and $t_{1 / 2}$ the crystallization half time, $\rho_{\mathrm{c}}$ and $\rho_{\mathrm{a}}$ are the densities of the crystalline and amorphous phases, respectively, and $X_{\mathrm{t}}$ stand for the maximum conversion. The results obtained suggest that the number of nuclei $(N)$ grows with increasing $\mathrm{PPz}$ content in the blend, as a consequence of a greater presence of $\mathrm{PPz}$ content in the PEO phase. When crystallization takes place in the presence of $\mathrm{PECH}, N$ increases considerably, due to the nucleating influence of PECH. In the case of ternary blends, an enormous increase of $N$ is noted for the blend PEO/PPz/PECH (20/60/20), owing to the fact that there coexist two nucleating actions, that of $\mathrm{PECH}$ and that of PPz. 
Parallelly a decrease in nucleating energy $\left(K_{\mathrm{g}}\right)$ is produced, as a function of $\mathrm{PPz}$ increase in the blend, i.e., the opposite effect of the one observed in the PECH systems, where $K_{\mathrm{g}}$ increases.

\section{COMPATIBILITY STUDY}

With the aim of studying the compatibility of the binary systems $\mathrm{PEO} / \mathrm{PPz}$ and $\mathrm{PEO} /$ $\mathrm{PECH}$, as well as the compatibilizing effect of $\mathrm{PECH}$ when incorporated into the incompatible binary system $\mathrm{PEO} / \mathrm{PPz}$, both the glass transition temperature and the Flory-Huggins interaction parameters were determined following the crystalline polymer melting point depression method, when in the presence of a diluting polymer.

The results of $T_{\mathrm{g}}$ analysis, as obtained from the DSC thermograms of the systems under study are compiled in Table VIII. For the system $\mathrm{PEO} / \mathrm{PECH}$ a single $T_{\mathrm{g}}$ is found, intermediate between those of the two polymers in isolation, which is indicative of total miscibility over the whole compositional range. The systems $\mathrm{PEO} / \mathrm{PPz}$ and $\mathrm{PPz} / \mathrm{PECH}$ each present two $T_{\mathrm{g}}$ values which remain practically constant independent of blend composition, as is characteristic of inmiscible systems. Nevertheless, in the ternary system there appear two transitions, the first of which can be attributed to the result of PEO/PECH compatibilization, and the second is the $T_{\mathrm{g}}$ of $\mathrm{PPz}$, which suggest the idea of partial compatibilization.

The interaction parameter was determined via the depression undergone by the melting point of crystalline polymers, when in the presence of a polymeric dilutant, according to the Nishi-Wang equation ${ }^{18-20}$ :

$$
\frac{1}{T_{\mathrm{m}}}-\frac{1}{T_{\mathrm{m}}{ }^{0}}=-\frac{R V_{2 \mathrm{u}}}{\Delta H_{2 \mathrm{u}} V_{1 \mathrm{u}}} X_{23} v_{1}{ }^{2}
$$

where $T_{\mathrm{m}}$ is the melting temperature, $X_{23}$ the Flory-Huggins interaction parameter, $V_{\mathrm{u}}$ represents the molar values and the subscripts 1 and 2 identify the amorphous and the crystalline polymers, respectively; $v_{1}$ is the volume fraction of the non-crystallizable component in the blend, and $T_{\mathrm{m}}{ }^{0}$ is the thermodynamic melting temperature obtained by applying the Hoffman-Weeks method ${ }^{17}$ (Figures 8 and 9). The values taken from the literature ${ }^{20}$ were as follows: $V_{2 \mathrm{u}}=39.2 \mathrm{~cm}^{3}$ $\mathrm{mol}^{-1}, \Delta H_{2 \mathrm{u}}=2100 \mathrm{cal} \mathrm{mol}^{-1}, V_{\mathrm{PECH}}=47.43$ $\mathrm{cm}^{3} \mathrm{~mol}^{-1}, V_{\mathrm{PPz}}=220.59 \mathrm{~cm}^{3} \mathrm{~mol}^{-1}$. From eq 6 the interaction parameters of the binary and ternary systems were computed as follows: $X_{23}=-0.172$ for the $\mathrm{PEO} / \mathrm{PECH}$ blend, a typically negative value of a compatible system; $X_{23}=0.032$ for $\mathrm{PEO} / \mathrm{PPz}$, positive and ap-
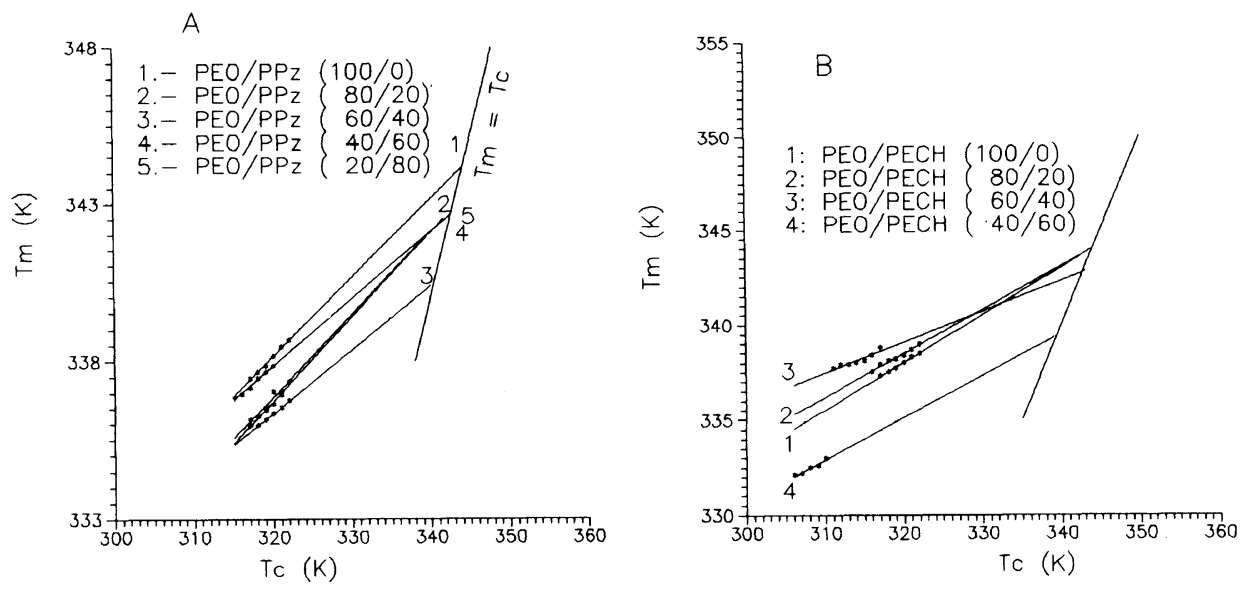

Figure 8. Hoffman-Weeks plots for A) PEO/PPz blend and B) PEO/PECH blend. 


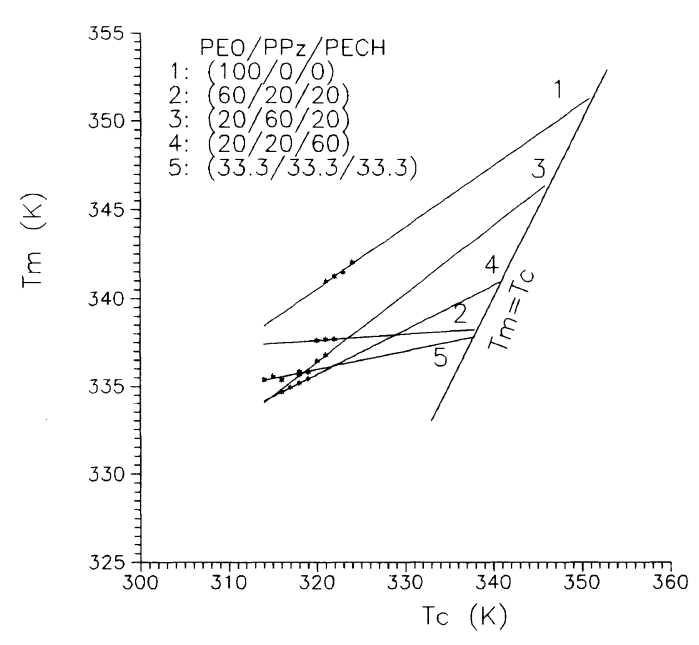

Figure 9. Hoffman-Weeks plots for $\mathrm{PEO} / \mathrm{PPz} / \mathrm{PECH}$ blend.

proaching zero, thus pointing toward the existence of incompatibility. Finally for the ternary blend $\mathrm{PEO} / \mathrm{PPz} / \mathrm{PECH}$ the value $X_{23}=-0.183$ was negative, identifying a compatible system.

By way conclusion and in the light of our data it is not unreasonable to consider that there exists sufficient evidence to confirm the remarkably (although not totally) compatibilizing effect exerted by PECH on the blend $\mathrm{PEO} / \mathrm{PPz}$, an effect which is enhanced by increasing the $\mathrm{PECH}$ portion in the blend.

Acknowledgments. Financial support of this work by the "Comunidad Autonoma de Madrid" of Spain (Project C076/91) is gratefully acknowledged.

\section{REFERENCES}

1. C. R. Herrero and J. L. Acosta, Polymer Int., in press.

2. M. Avrami, J. Chem. Phys., 7, 1103 (1939).

3. T. Ozawa, Polymer, 12, 150 (1971).

4. A. Ziabicki, Appl. Polym. Symp., 6, 1 (1967).

5. M. Mihailov, E. Nedkov, and I. Goshev, $J$. Macromol. Sci., Phys. Ed., 15, 313 (1978).

6. R. C. Allen and L. Mandelkern, J. Polym. Phys. Ed., 20, 1465 (1982).

7. E. Martuscelli, Polym. Eng. Sci., 24, 563 (1984).

8. G. C. Alfonso and T. P. Russell, Macromolecules, 19, 1143 (1986).

9. S. Cimmino, E. Martusccelli, C. Silvestre, M. Canetti, C. Lalla, and A. Seves, J. Polym. Sci., Polym. Phys., 27, 1781 (1989).

10. D. Turnbull and T. C. Fisher, J. Chem. Phys., 17, 71 (1949).

11. G. C. Alfonso and T. P. Russell, Macromolecules, 19, 1143 (1986).

12. J. D. Hoffman, Soc. Plast. Eng. J., A-2, 6, 885 (1968).

13. L. Mandelkern, "Crystallization of Polymers," McGraw Hill, New York, N.Y., 1964, p 273.

14. K. A. Min, J. S. Chion, J. W. Barlow, and D. R. Paul, Polymer, 28, 1721 (1987).

15. L. M. Robenson, W. F. Hale, and C. N. Merriam, Macromolecules, 14, 1644 (1981).

16. A. C. Fernandes, J. W. Barlow, and D. R. Paul, $J$. Appl. Polym. Sci., 29, 1971 (1984).

17. J. D. Hoffman and J. J. Weeks, J. Res. Natl. Bur. Stand., A66, 13 (1962).

18. T. Nishi and T. T. Wang, Macromolecules, 8, 809 (1975).

19. R. S. Stein, J. Polym. Sci., Polym. Phys. Ed., 20, 2243 (1982).

20. D. W. Van Krevelen, "Properties of Polymers," 2nd ed, Elsevier Scientific, Amsterdam, 1976. 